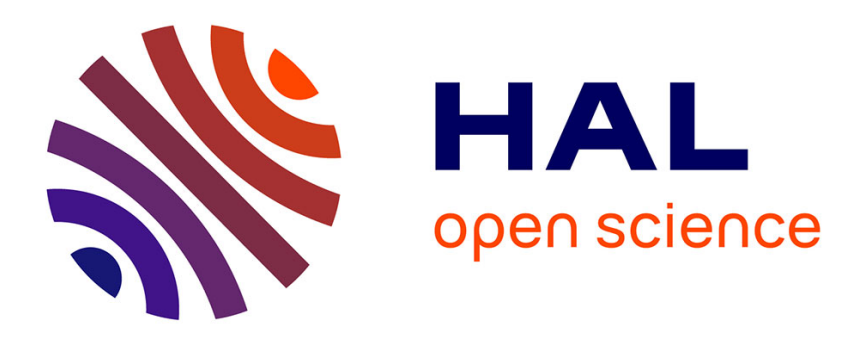

\title{
Modèles récents pour la propagation du son dans les matériaux poreux
}

\author{
J.-F. Allard
}

\section{To cite this version:}

J.-F. Allard. Modèles récents pour la propagation du son dans les matériaux poreux. Journal de Physique IV Proceedings, 1994, 04 (C5), pp.C5-177-C5-182. 10.1051/jp4:1994533 . jpa-00253028

\section{HAL Id: jpa-00253028 https://hal.science/jpa-00253028}

Submitted on 1 Jan 1994

HAL is a multi-disciplinary open access archive for the deposit and dissemination of scientific research documents, whether they are published or not. The documents may come from teaching and research institutions in France or abroad, or from public or private research centers.
L'archive ouverte pluridisciplinaire HAL, est destinée au dépôt et à la diffusion de documents scientifiques de niveau recherche, publiés ou non, émanant des établissements d'enseignement et de recherche français ou étrangers, des laboratoires publics ou privés. 


\section{Modèles récents pour la propagation du son dans les matériaux poreux}

\section{J.-F. ALLARD}

Laboratoire d'Acoustique de l'Université du Maine, URA 1101 du CNRS, Université du Maine, Avenue Olivier Messiaen, BP. 535, 72017 Le Mans cedex, France

Résumé : The modelling of the interactions between porous structures and saturating fluids has known new developments. D.L. Johnson has given a rigorous definition of tortuosity and has introduced a new parameter, the viscous characteristic dimension. A thermal characterictic dimension can be defined in a similar way. For the case of elastic structures, the two compressional waves must be taken into account. A matrix formulation is presented, which simplifies the modelling of stratified porous materials having an elastic structure.

\section{INTRODUCTION}

Parmi d'autres champs d'applications, les matériaux poreux sont utilisés dans les moyens de transport et les habitations pour de multiples usages, parfois comme isolants thermiques, et souvent comme absorbants acoustique. Une bonne connaissance de la propagation des ondes sonores peut permettre de mieux maîtriser leur utilisation.

Les matériaux poreux qui font l'objet de nos études sont constitués d'une structure solide baignant dans l'air. La porosité est le rapport entre le volume d'air et le volume total de matériau. Seule est prise en compte la porosité libre, associée dans le cas d'une mousse à des bulles ouvertes. La structure et l'air interagissent de différentes façons. Il y a des forces dues à la viscosité, et il y a aussi des effets inertiels, la tortuosité. Enfin les échanges thermiques entre l'air et la structure modifient l'incompressibilité de l'air.

La description microscopique de ces interactions est en général impossible, aussi les acousticiens ont ils d'abord étudié une structure simple, le pore cylindrique à section circulaire

\section{LE PORE CYLINDRIQUE A SECTION CIRCULAIRE}

On reprend brièvement l'étude de cette géométrie pour définir quelques notions importantes. Soit $v(r)$ le champ de vitesse des molécules d'air crée par un gradient de pression $\partial p / \partial z$ sinusoïdal dans la direction $\mathrm{Oz}$ de l'axe du pore. La vitesse, parallèle à l'axe, dépend de la distance $\mathrm{r}$ à l'axe. Elle est nulle sur les parois à cause de la viscosite et maximale sur l'axe. La vitesse moyenne $\bar{v}$ est 


$$
\bar{v}=\left[\int_{0}^{R} v(r) 2 \pi r d r\right] / \pi R^{2}
$$

$R$ étant le rayon du pore

On peut écrire une équation de Newton reliant $\bar{v}$ au gradient de pression

$$
-\frac{\partial p}{\partial z}=j \omega \rho_{e f f} \bar{v}
$$

La masse volumique $\rho_{0}$ de l'air est remplacée par $\rho_{\text {eff }}$ pour tenir compte des forces visqueuses, qui est donnée par

$$
\rho_{\text {eff }}=\rho_{0} / F(\omega) \text {. }
$$

avec $F(\omega)=1-\frac{2}{s \sqrt{-j}} \frac{J_{1}(s \sqrt{-j})}{J_{0}(s \sqrt{-j})}$, et $s=\left(\frac{\omega \rho_{0} R^{2}}{\eta}\right)^{1 / 2}$,

$\eta$ étant la viscosité de l'air et $\omega=2 \pi x$ fréquence.

Pour calculer l'incompressibilité de l'air, il faut évaluer le variation de température sur une section droite crée par une variation périodique de pression. La température sera constante sur la périphérie, car imposée par la structure, et présente des variations maximales sur l'axe. L'incompressibilité $\mathrm{K}$ est donnée par

$$
\mathrm{K}=\gamma \mathrm{P}_{0} /\left[\gamma-(\gamma-1) \mathrm{F}\left(\mathrm{B}^{2} \omega\right)\right]
$$

où $\gamma=c_{p} / c_{v}$ est le rapport des chaleurs spécifiques, $B^{2}$ est le nombre de Prandtl et $\mathrm{P}_{0}$ est la pression atmosphérique.

Le nombre d'onde complexe pour la propagation dans le tube sera

$$
\mathrm{k}=\omega\left(\rho_{\mathrm{eff}} / \mathrm{K}\right)^{1 / 2}
$$

Pour un matériau poreux à pores cylindriques circulaires identiques, on peut calculer le rayon $\mathrm{R}$ à partir de la résistance spécifique au passage de l'air, qui est un paramètre très important.

On soumet une couche de matériau poreux à un différentiel de pression $\mathrm{p}_{1}-\mathrm{p}_{2}$. Soit $\mathrm{V}$ le flux d'air qui traverse et e son épaisseur. La résistance au passage de l'air est la quantité

$$
\sigma=\left(p_{1}-p_{2}\right) /\left(V_{e}\right)
$$

Elle est reliée à $\mathrm{R}$ par

$$
\mathrm{R}=(8 \eta /(\sigma \phi))^{1 / 2}
$$

ou $\phi$ est la porosité. 
Le couple $(\phi, \sigma)$ suffit à caractériser le matériau.

\section{POREUX A GEOMETRIE DE PORE QUELCONQUE ET A STRUCTURE RIGIDE}

Il y a un nouveau paramètre, la tortuosité, et le rayon des pores est remplacé par 2 dimensions caractéristiques $\Lambda$ (pour les forces visqueuses) et $\Lambda^{\prime}$ pour les échanges thermiques.

\subsection{Tortuosité}

La définition rigoureuse de la tortuosité est récente et due à D.L. Johnson (Doll-Schlumberger Laboratories). Deux pores tortueux sont représentés en figure 1.

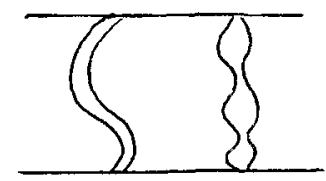

Figure 1 : Deux pores presentant de la tortuosité

La direction du vecteur vitesse des molécules d'air présente une dispersion dans le premier cas, et dans le second les étranglements créent une dispersion sur le module. La tortuosité $\alpha_{\infty}$ est liée à cette dispersion

$$
\alpha_{\infty}=v_{\text {microscopicque }}^{2}\left[\begin{array}{c}
\vec{v} \\
v
\end{array} \quad \alpha_{\infty}=v^{2} /(\overline{\bar{v}})^{2}\right.
$$

Pour éviter d'introduire la contribution de la viscosité à la dispersion, le fluide saturant n'est plus l'air mais un fluide idéal non visqueux. Compte tenu de l'analogie entre le champ de vitesse des ions dans un fluide conducteur saturant une couche poreuse, et celui des molécules de fluide non visqueux saturant la même structure, on peut évaluer la tortuosité à partir de mesures de résistivité. La tortuosité est donnée par:

$\alpha_{\infty}=\frac{\text { résistivité de la structure saturée d'un liquide conducteur }}{\text { résistivité du liquide conducteur }} \times$ porosité

\subsection{Dimensions caractéristiques}

Pour des pores circulaires, en hautes fréquences, $\rho$ et $\mathrm{K}$ peuvent être approximés par :

$$
\begin{aligned}
& \rho_{\text {eff }}=\rho_{0}\left[1+(1-\mathrm{j}) \frac{\delta}{\mathrm{R}}\right] \\
& \mathrm{K}=\gamma \mathrm{P}_{0} /\left[1+(\gamma-1)(1-\mathrm{j}) \frac{\delta}{\mathrm{BR}}\right],
\end{aligned}
$$


où $\delta$ est l'épaisseur de couche limite,

$$
\delta=\left(\frac{\eta}{\omega \rho_{0}}\right)^{1 / 2}
$$

et "hautes fréquences" signifie $\delta \ll R$.

La faible différence entre $\rho_{\text {eff }}$ et $\rho_{0}$ et $\mathrm{K}$ et $\gamma \mathrm{P}_{0}$, s'interprète par le fait que l'effet de la viscosité (ou des échanges thermiques) se manifeste sur une petite épaisseur autour de la paroi du tube d'ordre et de grandeur $\delta$. Johnson a généralisé l'expression de $\rho_{\text {eff }}$ au cas d'une structure quelconque en hautes fréquences.

$$
\rho_{\text {eff }}=\rho_{0} \alpha_{\infty}\left[1+(1-j) \frac{\delta}{\Lambda}\right]
$$

où $\Lambda$ est la dimension caractéristique visqueuse.

De même dans l'expression de $K, R$ peut ètre remplacé par une dimension caractéristique $\Lambda^{\prime}$. Les quantités $\Lambda$ et $\Lambda^{\prime}$ sont calculables assez rarement dans les cas réels, des procédés de mesure directe sont en cours d'élaboration au Mans.

\subsection{Expressions universelles pour la densité et l'incompressibilité}

Il existe des conditions asymptotiques en HF liées à $\Lambda$ et $\Lambda^{\prime}$ pour $\rho_{\text {eff }}$.

En BF il existe aussi des conditions asymptotiques. On doit retrouver la résistance au passage de l'air comme force visqueuse aux basses fréquences, et l'incompressibilité doit être isotherme car la structure impose sa température, fixe, à l'air. Johnson a donné une expression pour $\rho_{\text {eff }}$ qui satisfait toutes les conditions asymptotiques $\mathrm{HF}$ et $\mathrm{BF}$.

$$
\rho_{\text {eff }}\left(\alpha_{\infty}, \Lambda, \phi, \sigma, \omega, \eta\right)
$$

( $\rho_{\text {eff }}$ dépend de la tortuosité, la dimension visqueuse, la porosité, la résistance au passage de l'air, la viscosité)

De même, on peut chercher une expression universelle pour l'incompressibilité.

La plus simple est celle associée à un pore cylindrique circulaire de rayon $\Lambda^{\prime}$. Sur différents exemples ou $p_{\text {eff }}$ et $K$ ont été mesurés aux fréquences acoustiques, il y a bon accord entre prédictions et mesures. La connaissance de $\Lambda, \Lambda^{\prime}, \alpha_{\infty}, \sigma, \varnothing$ est suffisante pour caractériser la propagation du son dans les poreux à structure rigide.

\section{MATERIAUX POREUX A STRUCTURE SOUPLE}

Après des modèles entachés de beaucoup d'erreurs (Zwikker Kosten) ou incomplets (Beranek) est apparu un modèle semi-phénoménologique très élégant, la théorie de Biot. Ce modèle a été réactualisé par Johnson dars les années 80 , après la mise en évidence expérimentale par Plona des 2 modes de propagation longitudinaux prévus par la théorie. Nous ne pouvons pas valablement esquisser le modèle en quelques lignes, seulement quelques résultats importants sont indiqués. 


\section{Les ondes de Biot}

S'il n'y avait pas d'interaction entre l'air et la structure, 3 ondes de nature différente pourraient se propager dans un matériau poreux, une onde de compression dans l'air, et une onde de compression et une onde de cisaillement dans la structure considérée comme une solide élastique. Avec les interactions air-structure, 3 ondes subsistent. Aux fréquences acoustiques, pour les matériaux courants, les 2 ondes de structure gardent essentiellement les propriétés qu'elles présenteraient en l'absence d'interaction avec l'air, car l'air est beaucoup plus léger que la structure et la structure est insensible à la présence de l'air, même si l'air est ébranlé au passage de ces ondes. La troisième ondes de Biot est l'onde dans l'air profondément modifiée par l'interaction avec la structure. A son passage, la structure bouge très peu car elle est trop lourde.

Les ondes de structure sont en général négligées par les acousticiens Cela peut être justifiée dans le cas d'une couche poreuse posée sur une sol dur et soumise à un champ aérien. Cela n'est pas toujours vrai même dans ce cas. On a représenté en figure 2 l'impédance de surface d'une couche fibreuse posee sur un sol dur. La variation rapide de l'impédance autour de $400 \mathrm{~Hz}$ est due à la contribution de l'onde de compression de structure, associèe à une résonance quart d'onde. Dans beaucoup d'applications, les matériaux acoustiques sont des multicouches imperméables ou des écrans très résitifs. Une couche imperméables collée à une structure poreuse entraîne à le même vitesse la structure et l'air qui l'entoure, et les amplitudes des différentes ondes de Biot peuvent être comparables. La description de la propagation du son dans un multicouche avec 3 ondes aller et 3 ondes retour dans chaque couche ne présente pas de difficultés avec la représentation matricielle que nous avons développé au Mans dans la contexte de la théorie de Biot. Deux exemples de matériaux dont les propriétés acoustiques ne pourraient pas être modélisées correctement sans tenir compte des ondes de structure sont présentés en conclusion.

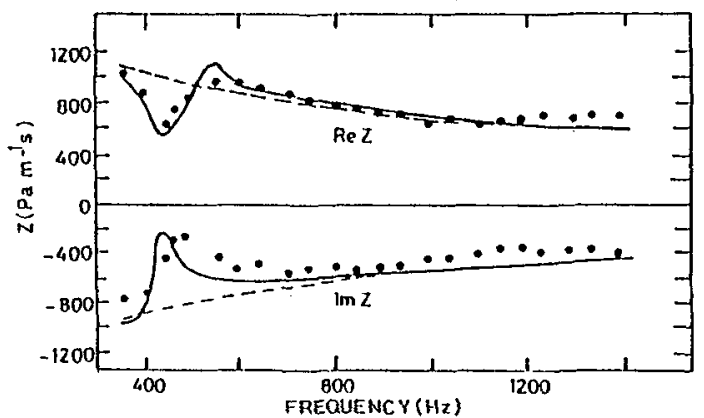

Figure 2 : Impédance d'une couche fibreuse ; mesure... Théorie de Biot Prédiction avec structure rigide - - -

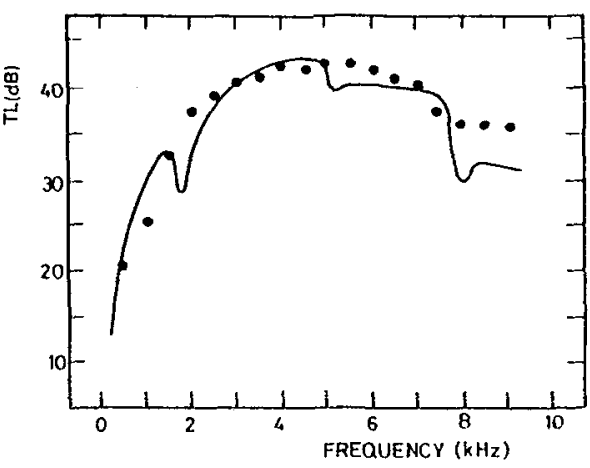

Figure $3:$ Transmission à travers une couche poreuse collée à une plaque métallique - champ diffus ; mesure $_{\text {owo }}$, théorie de Biot 


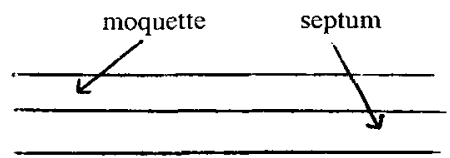

couche fibreuse

Figure 4 : Matériau stratifié pour l'absorption résonnante B.F.

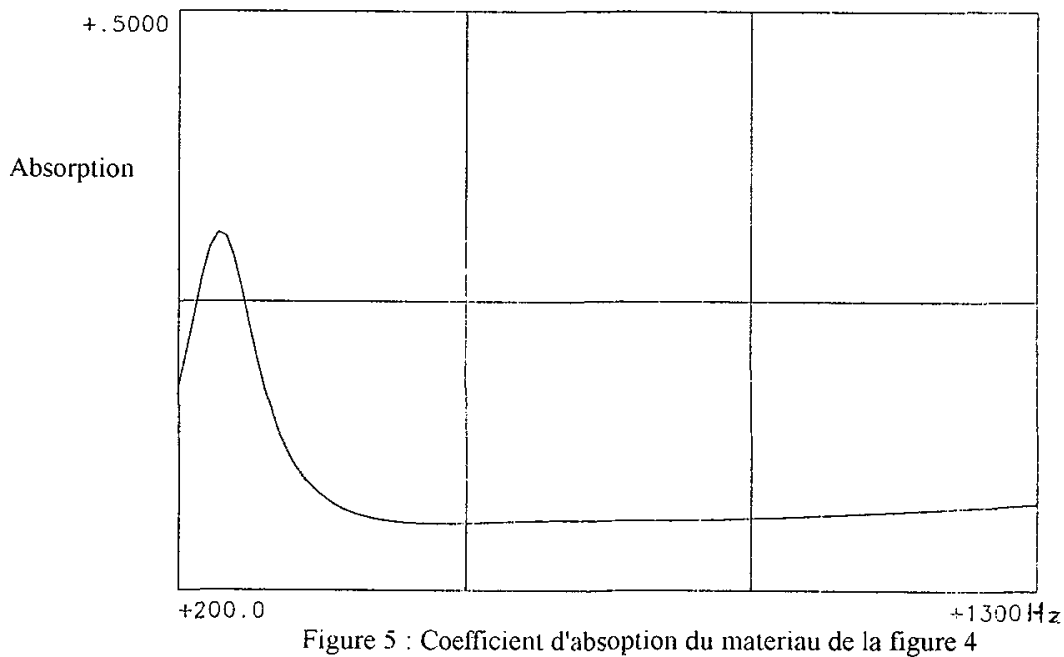

Le premier est un multicouche constitué d'un panneau fibreux collé à une plaque d'aluminuim. Ce type de structure est très utilisé en Aéronautique. Le coefficient de transmission en champ diffus est représenté en Figure 3, prédit avec la théorie de Biot et mesuré. Le second exemples est une multicouche représenté en Figure 4. Il est constitué d'une matériau fibreux sur lequel est placé un septum et une moquette. Le coefficient d'absorption de la moquette est très faible en basses fréquences. Le coefficient d'absorption (Figure 5), mesuré et prédit avec la theorie de Biot, est amélioré en basses fréquences grâce à une résonance du système septum - matériau fibreux.

\section{REFERENCES}

M.A. Biot, "The theory of propagation of elastic waves in a fluid - saturated porous solid", J. Acoust. Soc Am 28 (1956) 168 - 191

D.L. Johnson, J. Koplik, R. Dashen, "Theory of dynamic permeability and tortuosity in fluid saturated porous media",J. Fluid Mechanics 176 (1987) $379-402$

J.F. Allard, "Propagation of sound in porous media : Modelling sound absorbing materials", Chapman \& Hall, London (1993) 